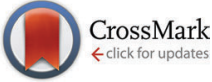

Cite this: Phys. Chem. Chem. Phys. 2016, 18, 22140

Received 13th June 2016, Accepted 14th July 2016

DOI: $10.1039 / c 6 c p 04099 c$

www.rsc.org/pccp

\title{
Ultrafast electron injection into photo-excited organic molecules $\nmid$
}

\author{
Dean Cvetko, ${ }^{\text {abc }}$ Guido Fratesi, ${ }^{\text {d }}$ Gregor Kladnik, $^{\text {ab }}$ Albano Cossaro, ${ }^{b}$ \\ Gian Paolo Brivio, ${ }^{e}$ Latha Venkataraman* ${ }^{* g}$ and Alberto Morgante ${ }^{\text {bh }}$
}

\begin{abstract}
Charge transfer rates at metal/organic interfaces affect the efficiencies of devices for organic based electronics and photovoltaics. A quantitative study of electron transfer rates, which take place on the femtosecond timescale, is often difficult, especially since in most systems the molecular adsorption geometry is unknown. Here, we use X-ray resonant photoemission spectroscopy to measure ultrafast charge transfer rates across pyridine/Au(111) interfaces while also controlling the molecular orientation on the metal. We demonstrate that a bi-directional charge transfer across the molecule/metal interface is enabled upon creation of a core-exciton on the molecule with a rate that has a strong dependence on the molecular adsorption angle. Through density functional theory calculations, we show that the alignment of molecular levels relative to the metal Fermi level is dramatically altered when a core-hole is created on the molecule, allowing the lowest unoccupied molecular orbital to fall partially below the metal Fermi level. We also calculate charge transfer rates as a function of molecular adsorption geometry and find a trend that agrees with the experiment. These findings thus give insight into the charge transfer dynamics of a photo-excited molecule on a metal surface.
\end{abstract}

\section{Introduction}

The performance of organic-based electronic devices relies on efficient charge transport across hybrid interfaces where electronically different materials couple; such interfaces often represent a bottleneck in the overall performance of organic electronic and photovoltaic devices. ${ }^{1}$ Charge transfer (CT) across interfaces is most easily studied using small aromatic molecules that can form ordered molecular films on a variety of substrates. Often, such systems exhibit rather uniform coupling schemes to metal electrodes as well as to other molecules, thereby minimizing structural disorder, which is a major limiting factor in the device performance. ${ }^{2,3}$ Such ordered prototypical systems open up the possibility to experimentally address charge transport phenomena on the molecular level using X-ray based spectroscopy techniques. ${ }^{4-7}$

\footnotetext{
${ }^{a}$ Faculty of Mathematics and Physics, University of Ljubljana, Slovenia. E-mail:dean.cvetko@fmf.uni-lj.si

${ }^{b}$ CNR-IOM, Laboratorio TASC, Basovizza, Trieste, Italy

${ }^{c} J$. Stefan Institute, Ljubljana, Slovenia

${ }^{d}$ ETSF and Dipartimento di Fisica, Università degli Studi di Milano, Italy. E-mail: guido.fratesi@unimi.it

${ }^{e}$ Dipartimento di Scienza dei Materiali, Università di Milano-Bicocca, Italy

${ }^{f}$ Department of Applied Physics, Columbia University, New York, NY, USA.

E-mail: lv2117@columbia.edu

${ }^{g}$ Department of Chemistry, Columbia University, New York, NY, USA

${ }^{h}$ Dipartimento di Fisica, Università di Trieste, Italy

$\dagger$ Electronic supplementary information (ESI) available. See DOI: 10.1039/c6cp04099c
}

Here we focus our study on films of pyridine molecules (PYR, $\mathrm{C}_{5} \mathrm{H}_{5} \mathrm{~N}$, inset, Fig. 1B) formed on $\mathrm{Au}(111)$ surfaces with adsorption geometries that can be experimentally varied by adjusting the molecular coverage. ${ }^{8}$ Our motivation for using this archetypical $\pi$-conjugated aromatic system is its ability to bind to $\mathrm{Au}$ surfaces through the formation of a weak $\mathrm{Au}-\mathrm{N}$ donor-acceptor bond, with geometry-dependent coupling, that has previously been shown to assist in charge transport through single-molecule conductance experiments. ${ }^{9}$ In this work, we use near
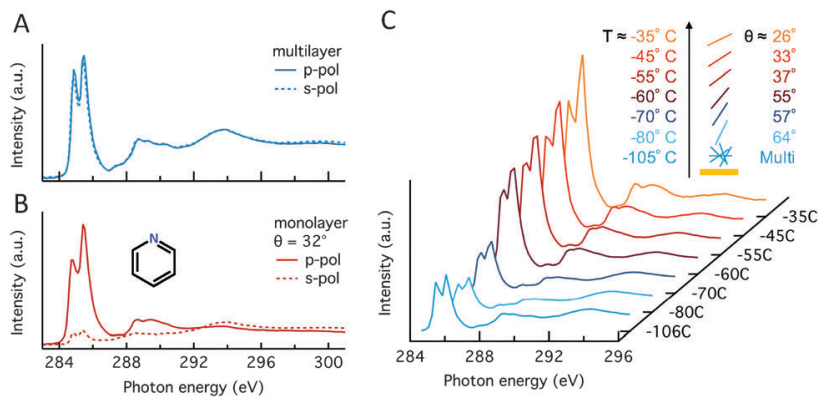

Fig. 1 C K-edge NEXAFS spectra of (A) a PYR multilayer film recorded at $-106{ }^{\circ} \mathrm{C}$ and (B) a monolayer film recorded at $-55^{\circ} \mathrm{C}$. The solid (dashed) lines are measurements with light polarized perpendicular (parallel) to the Au surface. Inset: PYR structure. (C) A series of C K-edge p-pol NEXAFS spectra of a multilayer film annealed up to $\sim-25{ }^{\circ} \mathrm{C}$ to create monolayers with different molecular orientations. All spectra have been normalized to the intensity at $295 \mathrm{eV}$. Note that temperatures and angles are approximate. 
edge X-ray absorption fine structure (NEXAFS), X-ray resonant photoemission (RPES) and apply core-hole-clock (CHC) analysis ${ }^{4}$ to study electron transfer between empty molecular orbitals and the Au continuum of states in the core-excited systems. The CHC method has been used previously to determine ultrafast electron transfer times from organic molecules to underlying surfaces, ${ }^{6,10-15}$ between aromatic molecules with $\pi-\pi$ coupling ${ }^{16}$ and also between molecules interacting through hydrogen bonding ${ }^{17}$ as well as between donor and acceptor organic molecules. ${ }^{18,19}$

Here, we present evidence for new core-hole decay channels present in the PYR-on-Au(111) system that reflect efficient injection of electrons from the Au substrate to PYR molecules on the femtosecond timescale, at a rate that depends on the orientation of the molecule on the Au substrate. We extend the standard CHC analysis to quantify the dynamics of electron injection to the molecule and find a strong dependence on the adsorption angle. The rate of electron injection from $\mathrm{Au}$ to the molecule increases as the molecular $\pi$ system becomes flatter, enabling a greater overlap with the Au substrate, in agreement with single-molecule transport measurements. ${ }^{9}$ Through density functional theory (DFT) calculations, we demonstrate that the core-excited lowest unoccupied molecular orbital (LUMO*) of the molecule lies partially below the Fermi level explaining the origin of the additional core-hole decay channels observed here. We also demonstrate that the coupling of the $\mathrm{LUMO}^{*}$ to the $\mathrm{Au}$ continuum states varies with the PYR adsorption angle in agreement with the experimentally observed trends.

\section{Results and discussion}

We first present results from near-edge X-ray absorption finestructure spectroscopy (NEXAFS) for PYR films on $\mathrm{Au}(111)$ and use them to determine the orientation of the molecule relative to the surface. ${ }^{8}$ We show, in Fig. 1A and B, NEXAFS spectra measured across the C K-edge over an energy range of 283-301 eV for a multilayer film and a monolayer film created by flash-heating a multilayer film to $-55{ }^{\circ} \mathrm{C}$ (see Fig. S1, ESI, $\dagger$ for additional details on film preparation and characterization through XPS). The main double-peak at $285 \mathrm{eV}$ corresponds to a photon absorption excitation from a C1s core level to the lowest unoccupied molecular orbital (LUMO). The double-peak feature results from different C1s initial states as presented in the ESI $\dagger$ with the help of DFT based calculations for an isolated molecule. The calculated NEXAFS spectra confirm that the LUMO can be excited from both $\mathrm{C}$ and $\mathrm{N}$ sites. The LUMO of PYR has $\pi^{*}$-character with its nodal plane coinciding with the molecular aromatic ring. ${ }^{9}$

We exploit the polarization dependence of the NEXAFS spectra, which are subject to dipole selection rules, to determine the molecular orientation on the surface. ${ }^{8}$ Specifically, the C1s $\rightarrow$ LUMO transition is forbidden for light polarized in the plane of aromatic rings. We therefore determine the average angle for PYR on $\mathrm{Au}(111)$ by comparing the intensity of this transition with an incident electric field perpendicular (p-pol) and parallel (s-pol) to the surface. For a multilayer film the intensity of the $\pi^{*}$ excitation is independent of the electric field polarization,
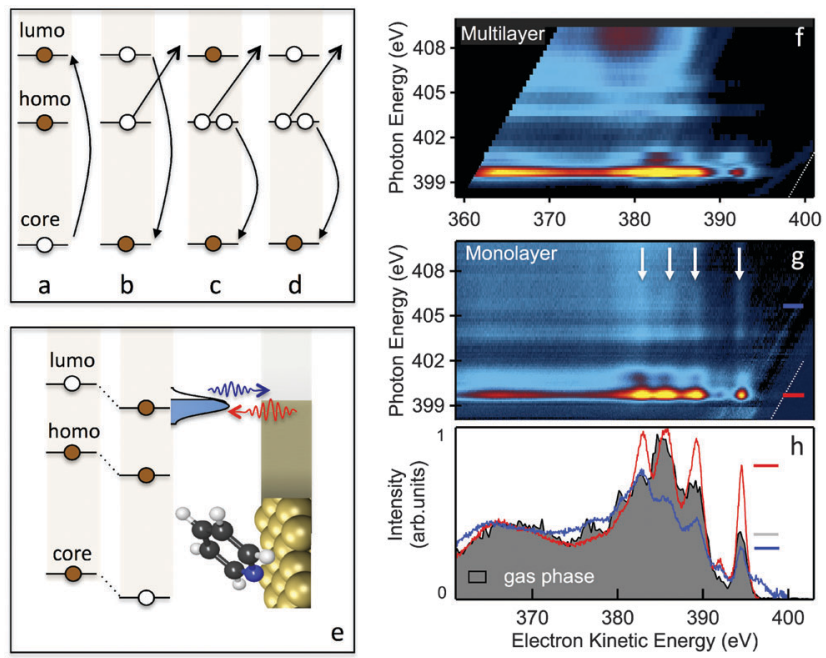

Fig. 2 Schematic of the RPES process: (a) core-hole/LUMO* excitation by $X$-ray absorption, (b) participator decay, (c) spectator decay and (d) normal Auger emission following LUMO* to substrate charge transfer. (e) Level alignment diagram following core-hole creation illustrating a bi-directional charge transfer as the LUMO* is aligned at the Fermi level, leading to the super-participator signatures in RPES maps. N K-edge RPES for ( $f$ ) multilayer film and (g) monolayer film oriented at $32^{\circ}$. The non-resonant photoemission intensity, measured below the $\mathrm{N} \mathrm{K}$-edge resonance (at $h \nu=395 \mathrm{eV}$ ) has been subtracted from all spectra. Arrows indicate the super-participator peaks due to ultrafast electron injection from the Au substrate. (h) Single RPES line scans at $h \nu=399.5 \mathrm{eV}$ and $405 \mathrm{eV}$ shown in red and blue, respectively. The grey shaded curve is the gas phase resonant spectrum at the N1s $\rightarrow$ LUMO excitation.

as can be expected for a randomly oriented molecular film. ${ }^{8}$ In contrast, the monolayer PYR film displays a strong linear dichroism with a higher intensity peak observed in p-pol compared to s-pol. For this film, the average molecular tilt angle $(\theta)$ from the surface, determined following previously published methods ${ }^{8}$ (see the ESI $\dagger$ ), is $32^{\circ} \pm 3^{\circ}$.

In Fig. 1C, we show a series of p-pol NEXAFS spectra collected while annealing a multilayer PYR film over a temperature range from $-106^{\circ} \mathrm{C}$ to $-25{ }^{\circ} \mathrm{C}$. The relative intensity of the C1s $\rightarrow$ LUMO peak increases gradually with increasing temperature indicating that the molecular orientation gets flatter upon annealing. The molecular adsorption angles obtained through heating a multilayer film range from $\sim 25^{\circ}$ to $\sim 65^{\circ}$.

We now turn to measurements of charge transfer dynamics at the PYR/Au interface by using the RPES technique and CHC analysis. As applied here, a core N1s electron is excited to an unoccupied molecular orbital (Fig. 2a) leaving a core-hole on the molecule. This excited state decays via three different scenarios.

In the first, known as the participator decay (Fig. 2b), the electron excited to the LUMO* de-excites in the core-hole decay with emission of an Auger electron. This leaves a single hole in one of the filled molecular orbitals and the LUMO empty. In the RPES spectrum these channels are found energetically degenerate with the respective direct photoemission from the filled molecular orbitals, i.e. HOMO $-n ; n=0,1,2,3$, etc., but with resonantly enhanced intensity. The second, known as spectator Auger decay (Fig. 2c), involves filling the core-hole with an electron from an occupied orbital, accompanied by electron emission from another 
occupied orbital; this leaves the system with two holes and an additional (spectator) electron in the LUMO. Finally, when the molecule is electronically coupled to the substrate, the excited LUMO* electron can escape to the substrate, quenching both the participator and spectator decay channels and the core-hole decays via a normal Auger process (Fig. 2d). By comparing the participator decay intensity in the monolayer and multilayer systems and knowing the core-hole lifetime ( 5 fs for N1s and 6 fs for C1s) ${ }^{20}$ we can determine the charge transfer times from the molecule to the substrate. However, another core-hole decay scenario arises if the LUMO* is lying below the Fermi level upon a core-hole creation (Fig. 2e). In this case, the LUMO* may get filled via electron transfer from the substrate. Thus, the characteristic spectral lines due to the decay of the LUMO* electron are observed regardless of the incident light energy; ${ }^{21,22}$ hereafter we term this process superparticipator decay.

We measure the RPES spectra, which are comprised of XPS measurements taken at a series of incident photon energies across the $\mathrm{N}$ K-edge of the PYR multilayer and 5 different monolayer films having PYR oriented with an angle ranging from $32^{\circ}$ to $56^{\circ}$. The experimental details are given in the ESI. $\dagger$ Briefly, XPS spectra over a $50 \mathrm{eV}$ kinetic energy range were measured with photon energy $(h \nu)$ tuned across the N K-edge in steps of $0.1 \mathrm{eV}$ (from $395 \mathrm{eV}$ to $415 \mathrm{eV}$ ) and all spectra are presented as two-dimensional maps of intensity plotted against photon energy and electron kinetic energy. Fig. 2f and g show RPES maps for a multilayer film and a monolayer film with molecules oriented at $32^{\circ}$. We see strong resonances in the RPES maps at $h \nu=399.5 \mathrm{eV}$, corresponding to an excited state decay following a N1s $\rightarrow$ LUMO* transition. These correspond to electron emission through the participator decay channel involving different occupied molecular orbitals at electron kinetic energies between 375 and $395 \mathrm{eV}$. Thus the photoemission spectrum on resonance $(h \nu=399.5 \mathrm{eV})$ resembles that of a direct valence band photoemission (see ESI, $\dagger$ Fig. S2) but with an enhanced intensity that depends on the spatial overlap of the occupied orbital and the LUMO with the N1s core orbital. ${ }^{6}$

We then analyze data of the PYR monolayer with an average molecular angle of $32^{\circ}$ relative to the Au surface. The monolayer RPES map (Fig. 2g) reveals additional features, marked by white arrows, not present in the multilayer map (Fig. 2f). These start at participator peaks in the spectrum at $h \nu=399.5 \mathrm{eV}$, and are visible even beyond excitations to the free electron continuum above $\sim 405 \mathrm{eV}$. These features are most clearly visible in the line scans shown in Fig. 2h, where we compare data at $h \nu=399.5 \mathrm{eV}$ and at $h \nu=405 \mathrm{eV}$ with a valence band spectrum acquired for the molecule in the gas phase. The clear peaks seen in the line scan taken at $405 \mathrm{eV}$ (beyond the ionization edge) indicate that there are additional features that occur at constant kinetic energy. We attribute these features to the super-participator process (Fig. 2e). Once a N1s core-hole is created in PYR, the energy level alignment of all orbitals relative to the metal Fermi level is altered. The LUMO* drops at least partially below the Fermi level and may get occupied with charge from the metal as long as it is electronically well-coupled to the substrate. Thus for all incident photon energies required to create a N1s core-hole $(h \nu \geq 399.5 \mathrm{eV})$, if an ultrafast charge injection into the LUMO* occurs within the core-hole life-time, the core-hole can decay through the super-participator channel.

Qualitatively we note that the intensity ratio of the superparticipator to participator peak is large and indicative of a fast electron injection into the molecule due to a significant fraction of the LUMO* lying below the Fermi level. To quantify the charge injection rate based on the data presented in Fig. 2, we measure the participator intensity, $I_{\mathrm{p}}$, at the LUMO* excitation $(h \nu=399.5 \mathrm{eV})$ and the super-participator intensity above the ionization edge, $I_{\mathrm{sp}}(h \nu=405 \mathrm{eV})$ for PYR on $\mathrm{Au}(111)$ and compare them with the participator intensity at the LUMO* excitation in the gas phase, $I_{\mathrm{p}}^{0}$. Details of the extended CHC analysis that use these peak intensities to determine charge transfer rates are given in the ESI. $\dagger$ For the PYR/Au system shown in Fig. 2 with a tilt angle of $32^{\circ}$, we measure $I_{\mathrm{sp}} / I_{\mathrm{p}}=0.7 \pm 0.05$ and $I_{\mathrm{p}} / I_{\mathrm{p}}^{0}=0.95 \pm 0.05$. This latter ratio already takes into account the angular dependence of the matrix element for the oriented molecules on the surface as $\cos (\theta) / \cos \left(\theta_{\mathrm{m}}\right)$, where $\theta_{\mathrm{m}}=54.7^{\circ}$ is used for the average over molecular orientations in the gas phase. This gives the fractional occupation of the LUMO* below Fermi $x \sim 1$, and for the electron injection time $\tau=\tau_{\mathrm{ch}}\left(1-I_{\mathrm{sp}} / I_{\mathrm{p}}\right) /\left(I_{\mathrm{sp}} / I_{\mathrm{p}}\right)=$ $3.2 \pm 0.5 \mathrm{fs}$, where $\tau_{\mathrm{ch}}=5 \mathrm{fs}$ is used for the N1s core-hole lifetime. ${ }^{20}$

We now repeat the RPES measurements and CHC analysis for four films with different molecular tilt angles ranging from $32^{\circ}$ to $56^{\circ}$. The results are summarized in Fig. 3. We find that the charge injection time increases from $\sim 3.2$ fs to $\sim 30$ fs with increasing tilt angle of the PYR molecules. In all cases we determine the fractional occupation of the $\mathrm{LUMO}^{*}$ to be close to unity $(x=0.9 \pm 0.1)$, indicating that LUMO* is filled by nearly one electron at all tilt angles of the adsorbed molecule.
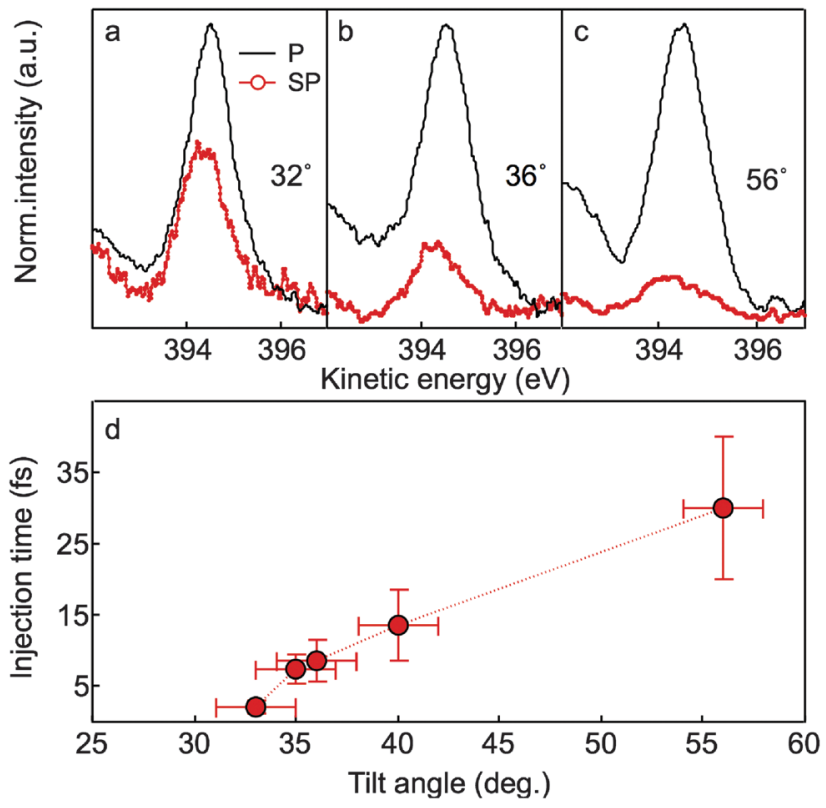

Fig. 3 Participator and super-participator peaks taken from line scans at $399.5 \mathrm{eV}$ and $405 \mathrm{eV}$ for the PYR monolayer with tilt angles of (a) $32^{\circ}$, (b) $36^{\circ}$ and (c) $56^{\circ}$. (d) The CT times obtained from CHC analysis of the participator and super-participator peaks as a function of molecular tilt angle. 
We also find a consistent trend of the Auger peak position above the ionization edge, passing from mostly "spectator shifted" to almost exclusively "normal Auger" for increasing tilt angles, in agreement with the observed trend in the charge injection times (details are shown in the ESI $\dagger$ ). These observations imply that the spatial overlap of LUMO* with the Au continuum of states is responsible for the strong angular dependence of the charge injection dynamics. We also find that ultrafast transfer of electrons from the Au occurs on both carbon and nitrogen sites of the core-excited molecule, and for both excitation sites flat lying adsorption geometries strongly enhance the rate of charge injection into the molecule.

In order to understand the effect of molecular adsorption geometry on the orbital level alignment in the presence of a core-hole and the influence of charge transfer across the organometallic interface, we turn to DFT calculations. For this work, we investigate five different molecular tilt angles: $\theta=0^{\circ}$, $30^{\circ}, 45^{\circ}, 60^{\circ}$, and $90^{\circ}$, and show in Fig. 4 a the computed density of states (projected on the LUMO of PYR) as a function of $\theta$, both for the ground state (blue bullets) and the N1s ionization (LUMO*, red triangles). To compare computational results with the experiment, we focus on the LUMO* case, with an added valence electron as eventually attracted from the substrate to screen the perturbation. ${ }^{23-27}$ This corresponds to a fully relaxed electronic configuration after the molecule is ionized. The attractive potential introduced by the core hole lowers the energy of the molecular orbitals with respect to the ground state. The LUMO*, in particular, shows a significant fraction going below the Fermi level (see red triangles in Fig. 4a) and hence being filled by electrons from the substrate, in agreement with the experimental observations, while the ground state
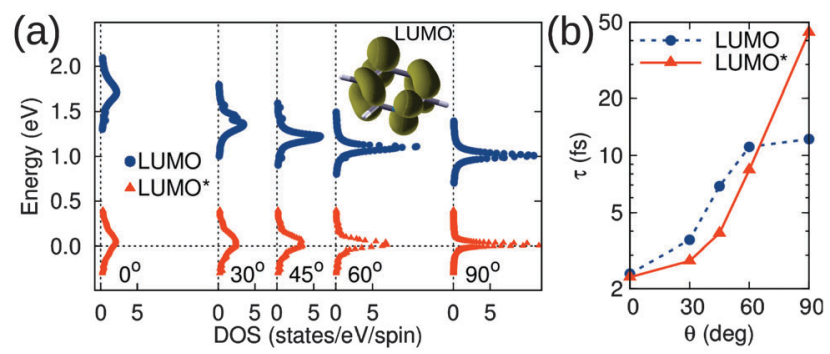

(c)

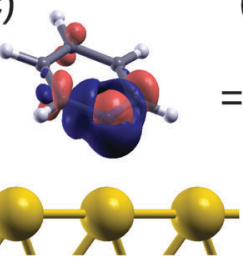

(d)

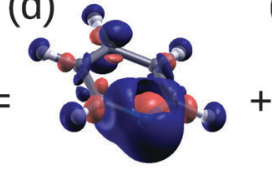

(e)

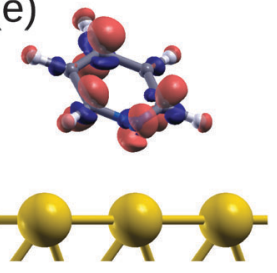

Fig. 4 (a) DFT DOS of PYR/Au(111) projected onto the LUMO of gas phase PYR (depicted in the inset) in the ground state (LUMO, bullets) and upon N1s $\rightarrow$ LUMO excitation (LUMO*, triangles). (b) Dependence of the resonant transfer time $\tau$ estimated by the LUMO/LUMO* width by a Lorentzian fit of the DOS, as a function of $\theta$. (c) Charge displacement $\Delta \rho=\rho_{\text {exc }}-\rho_{\text {gs }}$ for $\theta=45^{\circ}$. Regions of electron accumulation and depletion are colored in bright red and dark blue, respectively. (d) The same, for an isolated molecule in the gas phase: $\Delta \rho^{(\mathrm{g})}$. (e) Substrate contribution $\Delta \rho^{\prime}\left(\Delta \rho=\Delta \rho^{(\mathrm{g})}+\Delta \rho^{\prime}\right)$. The isodensity value in panels (d and e) is $0.04 \AA^{-3}$.
LUMO is always well above the Fermi level (blue bullets). The resonant transfer times for the LUMO and LUMO* are calculated from their Lorentzian width $\Gamma$, obtained for $\theta=45^{\circ}, \Gamma=95 \mathrm{meV}$ and $\Gamma=167 \mathrm{meV}$, i.e., $\tau=7 \mathrm{fs}$ and $\tau=4 \mathrm{fs}$, for the ground state and core-excited state, respectively. The difference between these values reflects the altered shape of the orbital as well as the interfacial energy-level alignment upon excitation. Although the computed value is smaller than the experimental one, the agreement is remarkable given the simplicity of the theoretical model.

We now consider the effect of the molecular adsorption angle on this charge transfer time. At variance with the moderate dependence found for the ground state LUMO, the energy alignment of the LUMO* is almost independent of $\theta$ so that electron transfer from the surface is expected at all angles considered. The $\pi$ symmetry of the orbital, which is depicted in the inset of Fig. 4a, is essential in determining the overlap with the substrate states and hence the dependence of $\tau$ on the angle. We observe a monotonous increase in the transfer time with $\theta$ (by a factor 3 when passing from $\theta=30^{\circ}$ to $60^{\circ}$ ), as shown in Fig. $4 \mathrm{~b}$, both for the values computed in the ground state and upon excitation, in agreement with the experimentally observed trend.

The electron transfer from the substrate to the ionized molecule can be visualized by evaluating the modification of the valence charge upon excitation, $\Delta \rho=\rho_{\text {exc }}-\rho_{\text {gs }}$. That is reported in Fig. $4 \mathrm{c}$ for $\theta=45^{\circ}$ and shows an increase of electron density in the molecular region: Mulliken population analysis ${ }^{28}$ performed in the ground and excited states show that about $0.89 e$ are transfered to the molecule. A similar result was obtained at the other angles, with increased charge transfer from $0.83 e$ to $0.93 e$ when passing from $\theta=0^{\circ}$ to $90^{\circ}$. These values are in excellent agreement with the experimental ones $(0.9 \pm 0.05)$. The remaining charge, $0.11 e$ for $\theta=45^{\circ}$, can be attributed to the Au atoms in the surface layer while the bottom layers are practically unaffected (we recall that $\rho_{\text {exc }}$ includes the added electron as provided by the metal). The electron displacement $\Delta \rho$ can be further decomposed into a molecular contribution $\Delta \rho^{(\mathrm{g})}$, as it would result for the ionization of free molecules in the gas phase (without the possibility of electron transfer), and a substrate contribution $\Delta \rho^{\prime}$ for electron transfer and screening also accounting for the additional valence electron: $\Delta \rho=\Delta \rho^{(\mathrm{g})}+\Delta \rho^{\prime}$. These quantities are shown in Fig. $4 \mathrm{c}, \mathrm{d}$ and e, respectively. While $\Delta \rho^{(\mathrm{g})}$ shows in Fig. $4 \mathrm{~d}$ a contraction of the molecular charge towards the $\mathrm{N}$ atom, the substrate contribution $\Delta \rho^{\prime}$ in Fig. 4e highlights the filling of the LUMO* state by surface electrons. We stress that these findings follow from the specific structure of $\Delta \rho$ of the PYR/Au system and may not occur generally for organic molecules adsorbed on metals. For example, a similar alignment of LUMO* to the Fermi level was computed for pyridine on graphene, ${ }^{27}$ but complete filling of LUMO* for pentacene/Al(001) has been evaluated and observed..$^{24,26}$

\section{Conclusions}

We explored the dynamics of charge transfer in a series of $\mathrm{PYR} / \mathrm{Au}(111)$ layers with different adsorption angles, and found 
that the LUMO* of the core excited molecules falls partially below the $\mathrm{Au}$ Fermi level, opening a gateway for ultrafast injection of electrons from the gold substrate to the molecule. Importantly, we have extended the standard CHC analysis of the RPES spectra to determine this charge transfer rate and have shown that it depends on the orientation of the molecule to the metal surface; the flatter molecules have a significantly faster charge transfer. Through DFT calculations, we have shown that the core-excited PYR/Au has a LUMO* that lies partially below the Fermi level, with an excellent agreement between computed occupation values and best-fit results from the measurements. We have also demonstrated that the broadening of this orbital due to its electronic coupling to the Au continuum of states enables an ultrafast charge transfer from the $\mathrm{Au}$ to the molecule within the core-hole lifetime. We have further calculated the angular dependence of the charge injection rate and find a trend consistent with the experiment of faster dynamics for flatter lying molecules. These findings have a direct consequence on understanding the mechanism of charge transfer at molecular junctions for hetero-organic photovoltaics, where the alignment of donor and acceptor orbitals in the presence of a valence exciton dictates the efficiency of exciton dissociation, charge separation, and consequently device performance.

\section{Methods}

Condensed phase and gas phase measurements have been performed at the ALOISA and ANCHOR beamlines of the Elettra Synchrotron in Trieste, Italy. ${ }^{29}$ The $\mathrm{Au}(111)$ substrate was prepared by cycles of $\mathrm{Ar}^{+}$ion sputtering and temperature annealing. Pyridine molecules were vacuum deposited on the Au substrate through a leak valve operated in $\sim 10^{-7}$ mbar pressure range. The PYR multilayer was grown with the sample held below $200 \mathrm{~K}$, whereas monolayer phases with variable adsorption geometry were produced by temperature annealing of multilayer films up to $T \sim 250 \mathrm{~K}$. NEXAFS at the nitrogen (carbon) K-edge was performed by collecting the emission of secondary electrons as a partial absorption yield with the high pass filter set to $370 \mathrm{eV}$ $(250 \mathrm{eV})$. All NEXAFS spectra were measured with linear photon polarization in s-pol geometry (electric field vector $\vec{E}$ parallel to the surface plane), p-pol geometry ( $\vec{E}$ along the surface normal) or at a magic angle (angle between $\vec{E}$ and the surface normal, $54.7^{\circ}$ ). Photoemission experiments were performed using an electron analyzer positioned along the photon polarization $\vec{E}$. Photon energies of $h \nu=650,500$, and $130 \mathrm{eV}$ were used for corelevel and valence band spectra, with an overall energy resolution of $\sim 100 \mathrm{meV}$. RPES at the N (C) K-edge was conducted by taking a series of XPS scans with photon energy between 395 and $425 \mathrm{eV}(280-310 \mathrm{eV})$ in steps of $0.1-0.2 \mathrm{eV}$. For each photon energy, the XPS spectrum covering $\sim 60 \mathrm{eV}$ kinetic energy $\left(E_{\mathrm{k}}\right)$ range was measured to construct a two-dimensional like RPES map $I\left(h \nu, E_{\mathrm{k}}\right)$. The non-resonant spectra were measured in the pre-edge region with a photon energy of $395 \mathrm{eV}(283 \mathrm{eV})$, and were subtracted from each XPS spectrum in the RPES maps $I\left(h \nu, E_{\mathrm{k}}\right)$. Participator intensity was evaluated after normalization

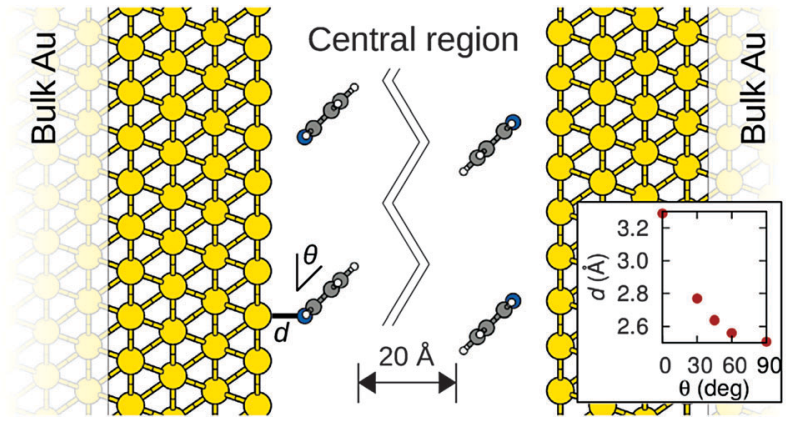

Fig. 5 Structural setup for computing the electronic structure of PYR adsorbed on semi-infinite $\mathrm{Au}(111)$. The inset shows the dependence of the $\mathrm{N}$-Au distance $d$ on the angle $\theta$ as determined in ref. 35.

to the integrated Auger, from the integrated intensities of the upper valence peaks (e.g. HOMO in the $0-8 \mathrm{eV}$ binding energy range). Super-participator intensity was evaluated from the XPS spectra with photon energy above the ionization edge, $h \nu>405 \mathrm{eV}$ $(h \nu>292 \mathrm{eV})$ as integrated intensity in the fixed kinetic energy range $(\sim 380 \mathrm{eV})$, containing the electron emission from the HOMO. More details about the spectra acquisition conditions, the preparation of the sample, and the implementation of the $\mathrm{CHC}$ method are given in the ESI. $\dagger$

We use the SIESTA/TranSIESTA simulation packages ${ }^{30,31}$ with the Perdew, Burke, and Ernzerhof (PBE) exchange-correlation functional. ${ }^{32}$ To obtain a detailed analysis of the coupling between the PYR molecule and the substrate, especially to deal with the lineshapes of the resonant orbitals, it is most convenient to consider a continuum of Au states. We adopt a description derived from electron transport ${ }^{31}$ where a semi-infinite substrate is taken into account by a Green's function approach. ${ }^{33,34}$ For computational convenience we replicate the system and model two facing semi-infinite $\mathrm{Au}$ substrates, each having a PYR molecular layer. Facing molecules are separated by at least $20 \AA$ to prevent any interaction between the two sides (see Fig. 5). Periodic boundary conditions in the lateral directions are taken within a $4 \times 4$ Au surface unit cell (coverage fixed to $1 / 16$ ).

In describing the angle-dependent properties, we adopt the molecular adsorption geometry on the $\mathrm{Au}(111)$ surface from the work of Mollenhauer et al., ${ }^{35}$ with the PYR N atom on the a-top site on the Au surface and an $\mathrm{Au}-\mathrm{N}$ distance that depends on the molecular tilt angle. Such a dependence was determined by structural optimizations within DFT corrected for van der Waals interactions, and is summarized in the inset of Fig. 5. To describe the case of a molecule excited by X-ray radiation, we promote a N1s electron from the core to a valence state. The core-hole is included in the pseudopotential that accounts for the interaction of valence electrons with a core-excited N1s* ion. The resonant electron/hole injection time $\tau$ from the LUMO is then determined as $\tau=\hbar / \Gamma^{36,37}$ from the Lorentzian full width at half-maximum (FWHM) $\Gamma$ in the density of states projected onto the LUMO:

$$
\rho_{\Phi}(E)=\frac{1}{\pi} \operatorname{Im}\left[G_{\Phi \Phi}(E)\right] \propto \frac{1}{\pi} \frac{(\Gamma+\gamma) / 2}{\left(E-E_{\Phi}\right)^{2}+[(\Gamma+\gamma) / 2]^{2}} .
$$


Here, the LUMO energy $E_{\Phi}$ and width $\Gamma$ are fitting parameters, $G_{\Phi \Phi}(E)$ is the expectation value of the Green's function on the $\Phi$ state, and $\gamma=4 \mathrm{meV}$ an additional broadening added for computational convenience.

\section{Acknowledgements}

The authors greatly acknowledge fruitful discussions with A. Batra and O. Adak. We acknowledge support from the MIUR of Italy through PRIN projects (no. 20104XET32 and 20105ZZTSE), ANCHOR project of the FIRB 2010 call (ref. RBFR10FQBL), and MAE project (US14GR12). Part of this research was also funded through Progetto Premiale 2012 ABNANOTECH. Computational resources are made available in part by CINECA (application codes HP10C0TP0R and HP10CESYLM). D. C. and G. K. acknowledge support from the Slovenian Research Agency (Project no. P1-0112 and Z1-6726). L. V. thanks the Packard Foundation for support.

\section{References}

1 S. R. Forrest, Nature, 2004, 428, 911-918.

2 F. Schreiber, Prog. Surf. Sci., 2000, 65, 151-257.

3 J. V. Barth, Annu. Rev. Phys. Chem., 2007, 58, 375-407.

4 P. A. Brühwiler, O. Karis and N. Mårtensson, Rev. Mod. Phys., 2002, 74, 703-740.

5 D. Menzel, Chem. Soc. Rev., 2008, 37, 2212-2223.

6 P. Vilmercati, D. Cvetko, A. Cossaro and A. Morgante, Surf. Sci., 2009, 603, 1542-1556.

7 L. Cao, X.-Y. Gao, A. T. S. Wee and D.-C. Qi, Adv. Mater., 2014, 26, 7880-7888.

8 J. Stöhr, NEXAFS Spectroscopy, Springer, Berlin, Heidelberg, 1992, vol. 25.

9 S. Y. Quek, M. Kamenetska, M. L. Steigerwald, H. J. Choi, S. G. Louie, M. S. Hybertsen, J. B. Neaton and L. Venkataraman, Nat. Nanotechnol., 2009, 4, 230-234.

10 L. Cao, Y.-Z. Wang, T.-X. Chen, W.-H. Zhang, X.-J. Yu, K. Ibrahim, J.-O. Wang, H.-J. Qian, F.-Q. Xu, D.-C. Qi and A. T. S. Wee, J. Chem. Phys., 2011, 135, 174701.

11 L. Cao, Y. Wang, J.-q. Zhong, Y.-Y. Han, W. Zhang, X. Yu, F. Xu, D. Qi and A. T. S. Wee, J. Phys. Chem. C, 2014, 118, 4160-4166.

12 G. Kladnik, D. Cvetko, A. Batra, M. DellâĂŹngela, A. Cossaro, M. Kamenetska, L. Venkataraman and A. Morgante, J. Phys. Chem. C, 2013, 117, 16477-16482.

13 J. Schnadt, P. A. Brühwiler, L. Patthey, J. N. O'Shea, S. Södergren, M. Odelius, R. Ahuja, O. Karis, M. Bässler, P. Persson, H. Siegbahn, S. Lunell and N. Mårtensson, Nature, 2002, 418, 620-623.

14 F. Blobner, P. B. Coto, F. Allegretti, M. Bockstedte, O. RubioPons, H. Wang, D. L. Allara, M. Zharnikov, M. Thoss and P. Feulner, J. Phys. Chem. Lett., 2012, 3, 436-440.

15 O. Adak, G. Kladnik, G. Bavdek, A. Cossaro, A. Morgante, D. Cvetko and L. Venkataraman, Nano Lett., 2015, 15, 8316-8321.
16 A. Batra, G. Kladnik, H. Vázquez, J. S. Meisner, L. Floreano, C. Nuckolls, D. Cvetko, A. Morgante and L. Venkataraman, Nat. Commun., 2012, 3, 1086.

17 G. Kladnik, M. Puppin, M. Coreno, M. de Simone, L. Floreano, A. Verdini, A. Morgante, D. Cvetko and A. Cossaro, Nano Lett., 2016, 16, 1955-1959.

18 P. Vilmercati, C. Castellarin-Cudia, R. Gebauer, P. Ghosh, S. Lizzit, L. Petaccia, C. Cepek, R. Larciprete, A. Verdini, L. Floreano, A. Morgante and A. Goldoni, J. Am. Chem. Soc., 2009, 131, 644-652.

19 T. Schiros, G. Kladnik, D. Prezzi, A. Ferretti, G. Olivieri, A. Cossaro, L. Floreano, A. Verdini, C. Schenck, M. Cox, A. a. Gorodetsky, K. Plunkett, D. Delongchamp, C. Nuckolls, A. Morgante, D. Cvetko and I. Kymissis, Adv. Energy Mater., 2013, 3, 894-902.

20 M. Coville and T. D. Thomas, Phys. Rev. A: At., Mol., Opt. Phys., 1991, 43, 6053-6056.

21 A. J. Britton, A. Rienzo, J. N. O'Shea and K. Schulte, J. Chem. Phys., 2010, 133, 094705.

22 C. Sauer, M. Wießner, A. Schöll, F. Reinert, M. WieBner, A. Schöll and F. Reinert, Mater. Sci., 2013, 17, 1-5.

23 S. García-Gil, A. García and P. Ordejón, Eur. Phys. J. B, 2012, 85, 239.

24 A. Baby, G. Fratesi, S. R. Vaidya, L. L. Patera, C. Africh, L. Floreano and G. P. Brivio, J. Phys. Chem. C, 2015, 119, 3624-3633.

25 T. Susi, D. J. Mowbray, M. P. Ljungberg and P. Ayala, Phys. Rev. B: Condens. Matter Mater. Phys., 2015, 91, 081401.

26 A. Baby, H. Lin, G. P. Brivio, L. Floreano and G. Fratesi, Beilstein J. Nanotechnol., 2015, 6, 2242-2251.

27 A. Ravikumar, A. Baby, H. Lin, G. P. Brivio and G. Fratesi, Sci. Rep., 2016, 6, 24603.

28 R. S. Mulliken, J. Chem. Phys., 1955, 23, 1833-1840.

29 L. Floreano, G. Naletto, D. Cvetko, R. Gotter, M. Malvezzi, L. Marassi, A. Morgante, A. Santaniello, A. Verdini, F. Tommasini and G. Tondello, Rev. Sci. Instrum., 1999, 70, 3855.

30 J. M. Soler, E. Artacho, J. D. Gale, A. Garca, J. Junquera, P. Ordejón and D. Sánchez-Portal, J. Phys.: Condens. Matter, 2002, 14, 2745-2779.

31 M. Brandbyge, J.-L. Mozos, P. Ordejón, J. Taylor, K. Stokbro and P. Ordejo, Phys. Rev. B: Condens. Matter Mater. Phys., 2002, 65, 1-17.

32 J. P. Perdew, K. Burke and M. Ernzerhof, Phys. Rev. Lett., 1996, 77, 3865-3868.

33 D. Sánchez-Portal, D. Menzel and P. M. Echenique, Phys. Rev. B: Condens. Matter Mater. Phys., 2007, 76, 235406.

34 G. Fratesi, C. Motta, M. I. Trioni, G. P. Brivio and D. SánchezPortal, J. Phys. Chem. C, 2014, 118, 8775-8782.

35 D. Mollenhauer, N. Gaston, E. Voloshina and B. Paulus, J. Phys. Chem. C, 2013, 117, 4470-4479.

36 S. Datta, Quantum Transport-Atom to Transistor, Cambridge University Press, 2005.

37 A. Nitzan, Chemical Dynamics in Condensed Phases: Relaxation, Transfer and Reactions in Condensed Molecular Systems, Oxford University Press, 2006. 